
ПАНКРЕАТИТОМ И РАКОМ ПОДЖЕЛУДОЧНОЙ ЖЕЛЕЗЫ

(с) И.Н. Григорьева ${ }^{1}$, О.В. Ефимова ${ }^{1 *}$, Т.С. Суворова ${ }^{2}$

'Федеральный исследовательский центр Институт цитологии и генетики Сибирского отделения Российской академии наук, Новосибирск, Россия;

${ }^{2}$ Новосибирский государственный медицинский университет, Новосибирск, Россия

Обоснование. В XXI в. в 2-3 раза выросла частота заболеваний поджелудочной железы (Пж) (острого панкреатита (ОП), хронического панкреатита (ХП) и рака поджелудочной железы (РПЖ)) наряду с увеличением их социально-экономической значимости. Ожирение, которое приняло масштабы пандемии, приводит к развитию целого ряда заболеваний. Результаты исследований о связи избыточной массы тела (МТ), ожирения с риском развития болезней Пж весьма неоднородны (для ОП и ХП) и немногочисленны (для ХП).

Цель. Определить частоту ожирения у больных ОП, ХП, РПЖ и сравнить эти показатели.

Методы. В ходе наблюдательного многоцентрового клинического кросс-секционного неконтролируемого исследования обследовано 44 больных ОП, 97 больных ХП и 45 больных РПЖ, группы были сравнимы по полу/возрасту. Письменное информированное согласие на участие в исследовании было получено от каждого пациента. Основной исход исследования: частота ожирения у больных ОП, ХП, РПЖ.

Результаты. Частота ожирения у больных ОП (13,6\%), ХП (24,7\%), РПЖ (20,0\%) достоверно не различалась. Среди обследованных больных наименьший средний индекс массы тела (ИМТ) $(24,2 \pm 0,7$ кг/м²) отмечен у больных ОП ( $p=0,049)$. Выявлена обратная связь ИМТ $\geq 22,5$ кг/м² с риском ОП (OR=0,398; 95\% ДИ 0,195-0,812; $p=0,011)$. У мужчин с ХП и у больных алкогольным ХП дефицит веса наблюдали чаще, чем у женщин с ХП и у больных билиарно-зависимым ХП соответственно. Показана обратная связь между ИМТ и «определенным» ХП (Ехр (В)=0,772; 95\% ДИ 0,632-0,942; $\mathrm{p}=0,011)$. Ранее (за год до настоящего обследования) ожирение чаще встречалось у больных РПЖ (55,6\%), чем у больных ОП (13,6\%; $\left.X^{2}=3,3 ; p=0,000\right)$ и XП (25,8\%; $\left.X^{2}=12,0 ; p=0,001\right)$. Показана прямая связь наличия ожирения в анамнезе (в нашем исследовании за год до обнаружения рака) с РПЖ $(\mathrm{OR}=4,435 ; 95 \%$ ДИ 2,180-9,025; $\mathrm{p}=0,000)$.

Заключение. Частота ожирения у больных ОП, ХП и РПЖ была сходной. У больных ХП средний ИМТ был выше, чем у больных ОП. ИМТ $\geq 22,5$ кг/м² являлся протективным фактором для ОП. ИМТ был обратно ассоциирован с «определенным» ХП. Наличие ожирения в анамнезе (за год до обнаружения РПЖ) было прямо связано с риском РПЖ.

КЛЮЧЕВЫЕ СЛОВА: острый панкреатит; хронический панкреатит; рак поджелудочной железы; ожирение.

\title{
THE FREQUENCY OF OBESITY IN PATIENTS WITH ACUTE PANCREATITIS, CHRONIC PANCREATITIS AND PANCREATIC CANCER
}

\author{
(c) Irina N. Grigor'eva', Olga V. Efimova1* ${ }^{1 *}$ Tatyana S. Suvorova²
}

${ }^{1}$ Federal Scientific Center Institute of Cytology and Genetics of the Siberian Branch of the Russian Academy of Sciences, Novosibirsk, Russia;

${ }^{2}$ Novosibirsk State Medical University, Novosibirsk, Russia

BACKGROUND: In the XXI century, the frequency of pancreas diseases increased 2-3 times. The expectation that causes a pandemic lead to the development of a number of diseases. The results of studies on the relationship of overweight, obesity with the risk of developing pancreas diseases (acute pancreatitis (AP), chronic pancreatitis (CP) and pancreas cancer (PC)) are very heterogeneous (for $\mathrm{AP}$ and $\mathrm{PC}$ ) and not numerous (for $\mathrm{CP}$ ).

AIMS: to identify the frequency of obesity in AP patients (APp), CP patients (CPp) and PC patients (PCp) and compare these parameters.

MATERIALS AND METHODS: at the observational multicenter clinical cross-sectional uncontrolled case-study $44 \mathrm{APp}, 97 \mathrm{CPp}$ and 45 PCp were examined; the groups were comparable by sex/age. Informed consent form for participate in the study was obtained from all patients. The main outcome of the study: the frequency of obesity in APp, CPp; PCp.

RESULTS: The frequency of obesity in APp (13,6\%), CPp (24,7\%) and PCp (20,0\%) did not differ significantly. Among the examined patients, the lowest average BMI $\left(24,2 \pm 0,7 \mathrm{~kg} / \mathrm{m}^{2}\right)$ was observed in APp $(p=0,049)$. BMI $\geq 22,5 \mathrm{~kg} / \mathrm{m}^{2}$ was found to be associated with $A P(O R=0,398 ; 95 \% C l 0,195-0,812 ; p=0,011)$. An inverse relationship was shown between the BMI and "definite" CP $(\operatorname{Exp}(B)=0,772 ; 95 \% C l 0,632-0,942 ; p=0,011)$. In men with CP and in CPp alcoholic etiology, weight deficit was observed significantly more often than in women with CP and in CPp biliary etiology, respectively. Earlier (a year before the present survey), obesity was more common in PCp (55,6\%) than in APp $\left(13,6 \%, X^{2}=3,3 ; p=0,000\right)$ and CPp $\left(25,8 \%, X^{2}=12,0\right.$; $p=0,001)$. A history of obesity (in our study one year before PC detection) and PC $(O R=4,435 ; 95 \% C l 2,180-9,025 ; p=0,000)$ direct relationship was shown. 
CONCLUSIONS: the frequency of obesity in APp, CPp and PCp was similar. The average BMI was higher in APp, than in CPp and PCp. BMI $\geq 22,5 \mathrm{~kg} / \mathrm{m} 2$ was a protective factor for AP. BMI was inversely associated with "defined" CP. A history of obesity was directly associated with PC.

KEYWORDS: acute pancreatitis; chronic pancreatitis; pancreatic cancer; obesity.

\section{ОБОСНОВАНИЕ}

Прогрессирующий рост заболеваемости, развития серьезных осложнений и смертности при остром панкреатите (ОП), хроническом панкреатите (ХП), раке поджелудочной железы (РПЖ) обуславливает необходимость углубленного исследования этих заболеваний. По данным BO3, в XXI в. отмечается тенденция к увеличению числа больных ОП молодого и среднего возраста, распространенность ХП в России за последние десятилетия выросла в три раза [1]. Ежегодно диагностируется около 338000 новых случаев РПЖ, среди причин онкологической смертности РПЖ занимает 4-е место в мире [1]. Люди с ожирением имеют повышенный риск развития полисистемной недостаточности органов при острых воспалительных состояниях [2]. При ожирении изменяется метаболическая активность жировой ткани - нарушается баланс адипокинов: повышается выработка провоспалительных адипокинов и угнетается выработка противовоспалительных, в том числе, и в поджелудочной железе (ПЖ) [1]. При этом сведения о связи между ожирением и заболеваниями ПЖ неоднородны.

Ожирение и ассоциированные с ним метаболические нарушения приводят к развитию целого ряда тяжелых заболеваний: дислипидемии, сахарного диабета 2 типа (СД2), желчнокаменной болезни (ЖКБ), неалкогольной жировой болезни печени (НАЖБП), злокачественным опухолям отдельных локализаций и др. [2, 3]. Эти заболевания, в свою очередь, способствуют увеличению риска ОП. Так, у больных ЖКБ выше вероятность развития панкреатита не только при помощи билиарного сладжа или микролитиаза, которые вызывают билиарно-панкреатический рефлюкс и запускают интрапанкреатическую активацию трипсина, но и за счет формирования метаболических нарушений [1]. Например, посредством гипертриглицеридемии (ГТГ), чаще отмечаемой при ЖКБ, СД2, НАЖБП, которая приводит к микротромбозу сосудов ПЖ, вызывающих ее ишемию и некроз, а также у больных с ГТГ-ассоциированным панкреатитом отмечается дефицит липопротеинлипазы (ЛПЛ) или аполипопротеина (аро) С и аро Е как лиганда для захвата печенью ТГ-богатых ремнантов [4]. Вследствие избытка циркулирующих триглицеридов происходит неконтролируемое высвобождение липотоксичных ненасыщенных жирных кислот (НЖК), которые увеличивают выброс провоспалительных цитокинов (фактор некроза опухоли (TNF-a), CXC-лиганда 1 (CXCL1) и CXCL2), что, в свою очередь, вызывает некроз ацинарных клеток, ингибируя митохондриальные комплексы I и V [5]. Однако в других исследованиях не обнаружена связь между ожирением и риском развития или тяжестью ОП [6].

Механизм ГТГ при СД2 связан с инсулинорезистентностью, что приводит к возврату избытка свободных жирных кислот (СЖК) в печень, с увеличением продукции липопротеинов очень низкой плотности (ЛПОНП) и снижением синтеза апоВ, и с гиперинсулинемией, которая способствует синтезу триглицеридов (ТГ) de novo, что в целом приводит к утяжелению течения ОП, однако другие авторы приводят доказательства того, что СД2 ассоциирован со снижением риска смерти от ОП в 1,22 раза по сравнению с больными ОП без СД2 [7].

В отличие от ОП, количество адипоцитов при ХП не связано с индексом массы тела (ИМТ): при ХП развивается выраженный фиброз ПЖ, и поскольку адипоциты, составляющие внутрипанкреатический жир, окружены массивными тяжами коллагеновых волокон, возникает препятствие для выхода липотоксичных СЖК, что предотвращает липолитический каскад между адипоцитами и ацинарными клетками, тем самым уменьшая ацинарный некроз [8]. В эксперименте установлено, что морфологическая структура ПЖ крыс при алиментарном ожирении имеет признаки продуктивного хронического воспаления [9], однако данные о коморбидности ожирения и ХП весьма немногочисленны.

Во многих исследованиях изучали ассоциации избыточной массы тела (ИзбМТ) и ожирения с риском развития РПЖ, но результаты этих исследований разноречивы: выявлена как прямая [10], так и опосредованная по полу связь между ожирением и РПЖ [11]. А при раке желудка, пищевода, легких выявлена обратная связь между ИМТ и смертностью [12]. В последнее десятилетие наблюдается «парадокс ожирения», когда у пациентов, страдающих раком, повышенный ИМТ связан с улучшением выживаемости по сравнению с пациентами с нормальным весом (ИМТ 22,5 кг/м² принят в качестве среднего ориентира для нормального веса) [13]. У онкологических больных с отдаленными метастазами показатели смертности были достоверно ниже, чем у пациентов с нормальным весом: в 1,2 раза ниже у больных с ИзбМТ и в 1,5 раза у пациентов с ожирением [12]. Кроме того, World Cancer Research Fund International (WCRFI) рекомендует определение ИМТ у онкологических больных до и после постановки диагноза [14]. Таким образом, разнородность сведений о связи ИзбМТ и ожирения с ОП, ХП и РПЖ, а также ограниченность данных по изучению этих показателей в рамках единого исследования побудили нас к выполнению настоящей работы.

\section{ЦЕЛЬ}

Определить частоту ожирения у больных ОП, ХП, РПЖ и сравнить эти показатели.

\section{МЕТОДЫ}

Дизайн исследования

Наблюдательное многоцентровое кросс-секционное неконтролируемое исследование по типу «серия случаев». Схема проведенного исследования представлена на рисунке 1. 


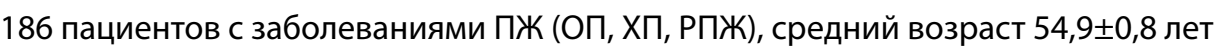

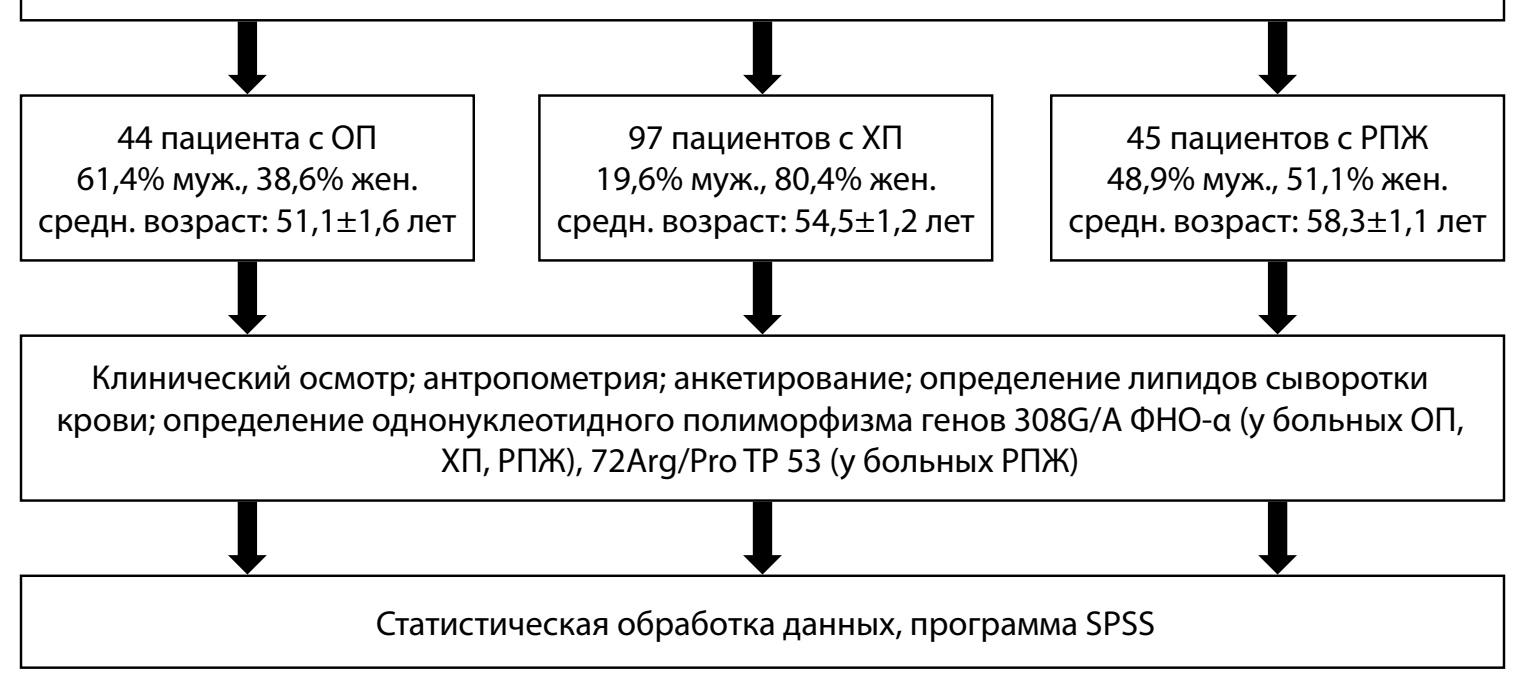

Рисунок 1. Дизайн исследования.

Примечания. ПЖ - поджелудочная железа, ОП - острый панкреатит, ХП - хронический панкреатит, РПЖ - рак поджелудочной железы, ФНО-а - фактор некроза опухоли-альфа.

\section{Критерии соответствия}

Критерии включения: больные ОП, ХП или РПЖ мужского и женского пола в возрасте 25-70 лет.

Критерии исключения: тяжелая сопутствующая патология.

\section{Условия проведения}

Поиск участников исследования проводился в лечебных учреждениях: стационаре Государственного бюджетного учреждения здравоохранения Новосибирской области «Государственная Новосибирская областная клиническая больница», Научно-исследовательском институте терапии и профилактической медицины - филиале Федерального государственного бюджетного научного учреждения «Федеральный исследовательский центр Институт цитологии и генетики Сибирского отделения Российской академии науки», дневном стационаре и консультативном диагностическом центре Государственного бюджетного учреждения здравоохранения Новосибирской области «Городская клиническая инфекционная больница № 1».

Продолжительность исследования

Исследование проводилось с 2014 по 2019 гг.

\section{Описание медицинского вмешательства}

Все больные заполняли опросники по степени выраженности клинических признаков болезней ПЖ, включая изменение МТ за предыдущий год до настоящего обследования или для РПЖ за год до выявления онкологии. Всем больным проведены антропометрические измерения. У всех больных проведен анализ анкетных данных и общеклинического обследования.

\section{Основной исход исследования}

Частота ожирения у больных ОП, ХП, РПЖ.

\section{Методы регистрации исходов}

Для верификации диагноза использовали общеклинические, лабораторные, инструментальные и морфологические методы обследования ПЖ [1]. Диагноз ОП установ- лен на основании сочетания минимум двух из следующих признаков при условии исключения другой хирургической патологии: типичная клиническая картина (интенсивные, не купируемые спазмолитиками боли опоясывающего характера, неукротимая рвота, вздутие живота; употребление алкоголя, острой пищи или наличие ЖКБ в анамнезе и др.); характерные признаки по данным ультразвукового исследования: увеличение размеров, снижение эхогенности, нечеткость контуров ПЖ; наличие свободной жидкости в брюшной полости; гиперферментемия (гиперамилаземия или гиперлипаземия), превышающая верхнюю границу нормы в три раза и более. Диагноз ХП установлен на основании комплексной оценки результатов проведенных инструментальных методов исследования ПЖ (ультразвукового исследования, компьютерной томографии или магнитно-резонансной томографии) и лабораторных тестов (определение уровней гликемии, эластазы-1 кала) с учетом критериев M-ANNHEIM. Определенный ХП выставлялся при наличии одного или нескольких из следующих критериев: кальцификация Пж, умеренные или тяжелые изменения протоков ПЖ, выраженная постоянная экзокринная недостаточность ПЖ. Вероятный ХП: легкие изменения протоков ПЖ, псевдокиста, патологические результаты функциональных тестов (эластазы-1 кала), эндокринная недостаточность. При наличии типичной клинической картины в отсутствие критериев вероятного и определенного ХП выставлялся диагноз «пограничный ХП» (Schneider A., 2007, [1]). Диагноз РПЖ заподозрен на основании данных инструментальных методов исследования, верифицирован морфологическим исследованием операционного материала. Оценка гистологических препаратов выполнена двумя патологами независимо с последующим обсуждением результатов и вынесением коллегиального заключения. При выявлении нейроэндокринного РПЖ диагностика новообразований проводилась в соответствии с критериями классификации опухолей эндокринных органов (ВО3, 2017).

Индекс массы тела Кетле II (ИМТ) вычисляли по фор-

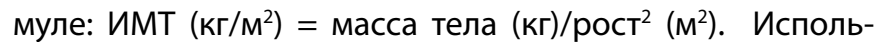
зовали классификацию ожирения по ИМТ (ВО3, 


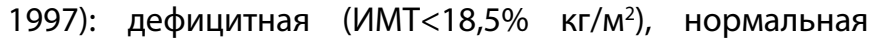
$\left(\right.$ ИМТ $=18,5-24,9$ кг $\left./ \mathrm{M}^{2}\right)$, ИзбМТ $(И М Т=25,0-29,9$ кг/м²), ожирение $\left(И М Т \geq 30 \mathrm{Kг} / \mathrm{M}^{2}\right)[2]$.

\section{Анализ в подгруппах}

Для анализа полученных результатов были выделены три группы: больные ОП, больные ХП и больные РПЖ. Группу ОП разделили на подгруппы по полу (мужчины и женщины), этиологии (алкогольный, билиарный, идиопатический) и по форме (отечный ОП, панкреонекроз). Группу больных ХП разделили на подгруппы по полу (мужчины, женщины), по этиологии (алкогольный, билиарно-зависимый, идиопатический) и по форме («определенный», «вероятный», «пограничный»). Группу больных РПЖ разделили на подгруппы по полу (мужчины, женщины), по форме (аденокарцинома ПЖ, нейроэндокринный РПЖ) и стадии (1-2 стадия и 3-4 стадия).

\section{Этическая экспертиза}

Исследование было одобрено этическим комитетом Научно-исследовательского института терапии и профилактической медицины - филиала Федерального государственного бюджетного научного учреждения «Федеральный исследовательский центр Институт цитологии и генетики Сибирского отделения Российской академии наук» (№ 38 от 23.09.14), Государственного бюджетного учреждения здравоохранения Новосибирской области «Городская клиническая больница № 7» (№1 от 31.03.2014), Государственного бюджетного учреждения здравоохранения Новосибирской области «Государственная Новосибирская областная клиническая больница» (№1 от 29.01.2014). Пациенты были проинформированы о возможном использовании их данных в научных целях. Все пациенты оставались анонимными при последующем анализе данных. Письменное добровольное информированное согласие на участие в исследовании было получено от каждого пациента.

Принципы расчета размера выборки. Предварительный расчет размера выборки не проводился.

\section{Статистический анализ}

Принципы расчета размера выборки: размер выборки предварительно не рассчитывался.

Методы статистического анализа данных: статистическую обработку данных проводили при помощи пакета программ SPSS (13.0). Проверку нормальности распределения признака проводили с использованием критерия Колмогорова-Смирнова. Сравнение частот качественных признаков проводилось с помощью критерия $\mathrm{X}^{2}$ и $\mathrm{t}$-критерия Стьюдента (t). Отношение шансов (OR) с 95\% доверительными интервалами (ДИ) рассчитывали по таблице сопряженности с поправкой Мантеля-Хенцеля. При сравнительной оценке средних значений количественных признаков исследуемых показателей между двумя группами применяли однофакторный дисперсионный анализ anova, между тремя группами - с поправкой в апостериорных множественных сравнениях Бонферрони. Результаты представлены как среднее арифметическое \pm ошибка среднего арифметического $(\mathrm{M} \pm \mathrm{m})$. Многомерный анализ связей главных признаков проводился с помощью логистического регрессионного анализа (метод Enter). Exp (В) (экс- понента В) отражает отношение рисков [15], показывает, во сколько раз изменяется риск возникновения исхода, если значение предиктора изменяется на единицу. Различия считались статистически значимыми при $\mathrm{p}<0,05$.

\section{РЕЗУЛЬТАТЫ}

Объекты (участники) исследования

В исследование включено 186 больных с заболеваниями ПЖ (ОП, ХП, РПЖ).

- 44 больных ОП: 61,4\% мужчин и 38,6\% женщин; средний возраст - 51,1 1,6 года.

- 97 больных ХП: 19,6\% мужчин и 80,4\% женщин; сред-

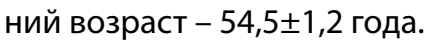

- 45 больных РПЖ, 48,9\% мужчин и 51,1\% женщин, средний возраст - 58,3 $\pm 1,1$ года.

В соответствии с классификацией ОП Савельева В.С. (2004) выявили 45,5\% больных с отечным ОП и 54,5\% больных с панкреонекрозом. По классификации M-ANNHEIM (Schneider A., 2007, [1]) в нашем исследовании больные ХП были распределены по клиническим формам: «пограничный» ХП - 45,36\%, «вероятный» ХП - 42,27\%, «определенный» ХП - 12,37\% и по этиологии - алкогольный ХП (17,5\%), билиарно-зависимый ХП (79,4\%), идиопатический (3,1\%). У 91,1\% больных РПЖ выявлена аденокарцинома ПЖ и у 8,9\% - нейроэндокринный рак. Распределение больных по стадии РПЖ: 57,8\% - Т1-Т2, 28,9\% - Т3 и 13,3\% - Т4 стадии.

Основные результаты исследования

Средние значения ИМТ, частота дефицитной, нормальной и ИзбМТ, а также ожирения у больных ОП, ХП и РПЖ представлены в таблице 1. Попарный анализ средних значений ИМТ у больных ОП, ХП и РПЖ выявил, что у больных ОП средний показатель ИМТ был значимо ниже, чем у больных ХП, а у больных РПЖ не отличался от такового у больных ОП и ХП. Кроме того, была выявлена обратная связь между ИМТ $\geq 22,5 \mathrm{kг} / \mathrm{M}^{2}$ и риском ОП (OR=0,398; 95\%ДИ 0,195-0,812; p=0,011). Логистический регрессионный анализ (с включением в зависимые переменные панкреонекроза, «определенного» ХП или 3-4 стадии РПЖ и в независимые - возраста, пола, ИМТ) выявил обратную связь ИМТ с «определенным» ХП $(\operatorname{Exp}(B)=0,772 ; 95 \% Д И ~ 0,632-0,942 ; p=0,011)$, то есть с повышением на 1 единицу ИМТ риск ХП снижался в 0,8 раза.

Частота дефицитной, нормальной, избыточной массы тела и ожирения у больных ОП, ХП и РПЖ значительно не различалась. Логистический регрессионный анализ с зависимыми (ОП, ХП и РПЖ) и независимыми переменными (возраст, дефицитная, нормальная, ИзбМТ, ожирение) не выявил достоверных ассоциаций этих заболеваний и МТ.

Учитывая среднюю распространенность ожирения в России $(29,7 \%)$ [16], у больных ОП ожирение $(13,6 \%)$ встречалось в 2 раза реже $(\mathrm{t}=2,3 ; \mathrm{p}=0,020)$, а частота ожирения у больных РПЖ (20,0\%; $t=1,4 ; p=0,155)$ и ХП $(24,7 \%$; $\mathrm{t}=1,1 ; \mathrm{p}=0,286)$ различалась незначительно. Среди обследованных больных не было обнаружено связи между ожирением и ОП (OR=0,522; 95\% ДИ 0,203-1,342; $\mathrm{p}=0,177)$, XП $(O R=1,622 ; 95 \%$ ДИ 0,788-3,327; $p=0,189)$ или РПж

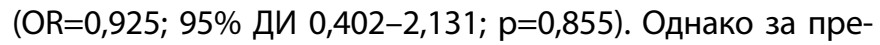
дыдущий год до настоящего обследования 68,2\% больных ОП, 45,4\% больных ХП и 84,4\% больных РПЖ снизили вес, 
Таблица 1. Характеристики массы тела у больных острым панкреатитом (ОП), хроническим панкреатитом (ХП) и раком поджелудочной железы (РПЖ)

\begin{tabular}{|c|c|c|c|c|c|c|}
\hline Группа больных & $0 \Pi, n=44$ & $\mathrm{Xn}, \mathrm{n}=97$ & PПЖ, n=45 & $\mathbf{P}_{\text {(опихп) }}$ & $\mathbf{p}_{\text {(оП и РПж) }}$ & $\mathbf{p}_{\text {(ХП и РПЖ }}$ \\
\hline 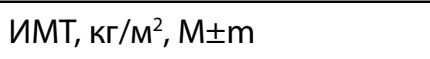 & $24,2 \pm 0,7$ & $26,3 \pm 0,5$ & $26,2 \pm 0,7$ & 0,049 & 0,158 & 1,000 \\
\hline Дефицитная МТ & $3(6,8 \%)$ & $5(5,2 \%)$ & - & 0,693 & - & - \\
\hline Нормальная МТ & $22(50 \%)$ & $37(38,1 \%)$ & $20(44,4 \%)$ & 0,188 & 0,601 & 0,477 \\
\hline Избыточная МТ & $13(29,5 \%)$ & $31(32 \%)$ & $16(35,6 \%)$ & 0,775 & 0,547 & 0,672 \\
\hline Ожирение & $6(13,6 \%)$ & $24(24,7 \%)$ & $9(20 \%)$ & 0,138 & 0,425 & 0,535 \\
\hline $\begin{array}{l}\text { Ожирение (за год до } \\
\text { настоящего обследования) }\end{array}$ & $6(13,6 \%)$ & $25(25,8 \%)$ & $25(55,6 \%)$ & 0,000 & 0,001 & 0,107 \\
\hline
\end{tabular}

Примечания. ИМТ - индекс массы тела; ОП - острый панкреатит; ХП - хронический панкреатит; РПж -рак поджелудочной железы. В таблице представлена сравнительная характеристика ИМТ, частоты дефицитной, нормальной, избыточной массы тела, ожирения. Различия считались статистически значимыми при $\mathrm{p}<0,05$.

$X^{2}=19,2 ; p=0,000$ при сравнении больных ХП и РПЖ, $X^{2}=3,3 ;$ $\mathrm{p}=0,071$ при сравнении больных ОП и РПЖ. Вес снизился

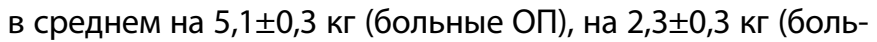
ные ХП) и на 14,0 $\pm 1,4$ кг (больные РПЖ); $p=0,000$ при сравнении больных РПЖ с больными ОП или ХП. Ранее (за год до настоящего обследования) ожирение чаще встречалось у больных РПЖ (55,6\%), чем у больных ОП (13,6\%; $\left.X^{2}=3,3 ; p=0,000\right)$ и XП $\left(25,8 \%, X^{2}=12,0 ; p=0,001\right)$. С учетом этих сведений была продемонстрирована связь ожирения в анамнезе (в нашем исследовании за год до обнаружения РПЖ) с РПЖ (OR=4,435; 95\%ДИ 2,180-9,025; $\mathrm{p}=0,000)$.

Характеристики MT практически не различались в зависимости от пола, этиологии ОП; пола, формы или стадии РПЖ. Среди больных ХП отмечены значимые гендерные различия: частота дефицитной МТ была выше у мужчин с ХП $(15,8 \%)$, чем у женщин с ХП $(2,6 \%)$ в 6 раз $\left(x^{2}=5,5 ; p=0,019\right)$, а ИзбМТ, наоборот, меньше в 7 раз (5,3 и 38,5\% соответственно; $\left.X^{2}=7,7 ; p=0,005\right)$. У больных билиарно-зависимым ХП средний показатель ИМТ был выше $(27,2 \pm 0,5$ кг/м²), чем

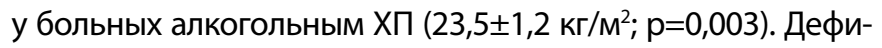
цит МТ чаще наблюдали у больных алкогольным ХП (17,6\%) по сравнению с таковым у больных билиарно-зависимым ХП (1,3\%), $X^{2}=9,1 ; p=0,003$. У больных «определенным» ХП средний показатель ИМТ был наименьшим $(22,0 \pm 1,6$ кг/M²) по сравнению с «вероятным» $(26,0 \pm 0,7$ кг/м²; $=0,024)$ и «по-

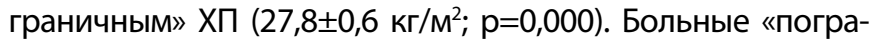
ничным» ХП в 5 раз чаще отмечали ИзбМТ, чем больные «определенным» $X П\left(X^{2}=11,6 ; p=0,001\right)$. Однако частота ожирения достоверно не различалась у больных «определенным» ХП (8,3\%), «вероятным» ХП (22,0\%) и «пограничным» ХП (31,8\%).

Логистический регрессионный анализ (с включением в зависимые переменные панкреонекроза, «определенного» ХП или 3-4 стадии РПЖ и в независимые - возраста, дефицитной МТ и ожирения) выявил прямую связь дефицитной МТ с «определенным» ХП $(\operatorname{Exp}(B)=14,706 ; 95 \%$ ДИ 1,903-113,647; $p=0,010)$.

\section{ОБСУЖДЕНИЕ}

\section{Резюме основного результата исследования}

В нашем исследовании частота ожирения значительно не различалась у больных ОП, ХП и РПЖ. При сравнении частоты ожирения со средней распространенностью ожирения в России (29,7\%) [16] получены данные о том, что среди больных ОП ожирение встречалось в 2 раза реже, частота ожирения у больных РПЖ и ХП значительно не различалась. Логистический регрессионный анализ не выявил связи ожирения с панкреонекрозом, «определенным» ХП или 3-4 стадиями РПЖ.

Обсуждение основного результата исследования

В ранее проведенных метаанализах показано, что ожирение является одним из наиболее важных негативных прогностических факторов ОП: увеличивает риски развития, коррелирует с тяжестью болезни, развитием локальных и системных осложнений [17-19]. В экспериментальном исследовании в модели на мышах ожирение также коррелировало с тяжестью ОП [20]. Тем не менее другие исследователи ставят под сомнение ассоциацию ожирения и ОП. В работе Hall (2015), наоборот, распределение жира (подкожное, забрюшинное и внутрибрюшное) у больных ОП не влияло на тяжесть и смертность от этого заболевания [21]. В японском исследовании Taguchi (2014) показано, что дефицит МТ, а не ожирение является негативным прогностическим признаком летальности ОП [22]. Этому не противоречат результаты нашего исследования: выявлена обратная связь ИМТ $\geq 22,5$ кг/м² с риском ОП. При обзоре литературных данных мы не обнаружили убедительных эпидемиологических доказательств ассоциации ожирения и риска развития ХП. В исследовании Ammann (2010) ИзбМТ и ожирение обнаружены в 54,2 и 15\% случаев алкогольного ХП соответственно, что было значительно выше по сравнению с контрольной группой $(37,7$ и 3,1\%) [23]. Машариповой (2019) определены у больных

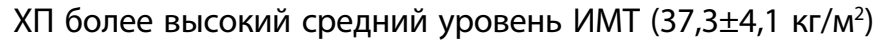
и высокую частоту ИзбМТ (75\%) [24], в другом исследовании, наоборот, отмечены более низкий средний ИМТ

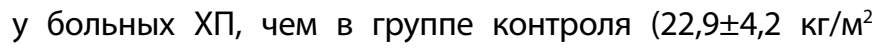

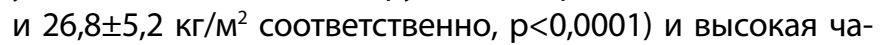
стота дефицитной МТ (26\%) [25]. В исследовании Roberts (2019) у больных ХП также определен более низкий ИМТ

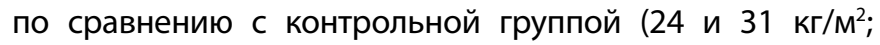
p<0,001) [26]. Кроме того, у больных ХП описаны случаи саркопенического и остеосаркопенического ожирения, когда снижение физической активности в результате саркопении приводит к увеличению жировой массы, а имеющееся ожирение усугубляет саркопению через снижение чувствительности мышц к инсулину, развившееся из-за повышения продукции провоспалительных цито- 
кинов, нарушения регуляции секреции лептина и адипонектина [27]. В работе Губергриц (2013) показано, что у больных ХП на фоне ожирения развиваются типичные для ХП структурные изменения ПЖ (увеличение размеров, неровность контуров, изменение эхогенности, кальцификация, расширение главного панкреатического протока, псевдокисты ПЖ) [28]. Данные о влиянии ожирения на риски РПЖ неоднозначны: в одних исследованиях обнаружена прямая связь между ожирением и РПЖ [29], в других - обратная связь [30]. Результаты объединенного анализа 20 перспективных когортных исследований Genkinger J. (2015) подтвердили связь между смертностью от РПЖ и центральным ожирением [31]. Shi (2016) провели метаанализ о прогностической роли ИМТ на выживание при РПЖ. Результаты показали, что ожирение связано с риском развития РПЖ $(\mathrm{OR}=1,29)$ и более низкой выживаемостью от РПЖ в зрелом возрасте (риск смерти от РПЖ повышался на $11 \%$ на каждые 5 единиц прироста ИМТ) [32]. В метаанализе 13 исследований Majumder (2016) продемонстрировали повышение смертности, связанной с РПЖ у лиц с ИзбМТ $(\mathrm{OR}=1,06)$ и ожирением $(\mathrm{OR}=1,31)$ по сравнению с контрольной группой. Каждое увеличение ИМТ на 1 кг/м² было связано с увеличением смертности на 10\% [33]. Согласно результатам Всемирного фонда исследования рака (2007), есть «убедительный повышенный риск» РПЖ у тучных лиц и «вероятный повышенный риск» у лиц с абдоминальным ожирением [34]. В нашем исследовании не выявлено связи наличия ожирения (на момент обследования) с развитием РПЖ, однако подтверждена ассоциация ожирения в анамнезе (за год до обнаружения РПЖ) с РПЖ (OR=4,4; 95\%ДИ 2,2-9,0; $\mathrm{p}=0,000)$. Тот факт, что ранее (до снижения веса, возможно, в результате онкогенеза) ожирение чаще встречалось у больных РПЖ $(55,6 \%)$ по сравнению с больными ОП (13,6\%; р<0,05) и ХП $(25,8 \%$; $\mathrm{p}<0,05)$, может служить косвенным доказательством более значительной роли ожирения при РПЖ, чем при воспалительных заболеваниях ПЖ. В настоящее время исследователи призывают шире использовать возможность модифицировать МТ с целью улучшения течения как воспалительных, так и онкологических заболеваний ПЖ.

\section{Ограничения исследования}

К ограничениям исследования следует отнести малое число наблюдений, неравномерное распределение по полу среди больных ХП (из 97 больных ХП 19,6\% мужчин и 80,4\% женщин). Кроме этого, в нашем исследовании не изучались такие показатели абдоминального ожирения, как окружности талии, бедер, отношение окружности талии к окружности бёдер.

\section{ЗАКЛЮЧЕНИЕ}

Во многих исследованиях показана ассоциация ожирения с риском ОП, тяжестью болезни и частотой осложнений. ХП относится к хроническим неинфекционным заболеваниям, однако влияние одного из основных факторов риска хронических неинфекционных заболеваний на ХП практически не изучено. Разноречивость данных по связи ожирения с риском РПЖ, вероятно, может быть объяснена недоучетом более высокой частоты ожирения до развития онкологии. Согласно отчету WCRF, определение ИМТ как до болезни, так и после постановки диагноза - важный момент в изучении влияния факторов риска на выживаемость при раке. В нашем исследовании среди обследованных больных только при ОП средний ИМТ соответствовал критериям нормальной МТ, при ХП и РПЖ - ИзбМТ. Ассоциации между различными характеристиками МТ и наличием ОП, ХП или РПЖ не было обнаружено. ИМТ $\geq 22,5$ кг/м² являлся протективным фактором для ОП. У мужчин с ХП дефицит веса встречался чаще, чем у женщин с ХП. Логистический регрессионный анализ выявил прямую связь дефицитной МТ с «определенным» ХП. Наличие ожирения в анамнезе (за год до обнаружения РПЖ) было прямо связано с риском РПЖ.

Таким образом, результаты нашего исследования не подтвердили ассоциации ожирения с ОП, что отличается от результатов метаанализов, проведенных ранее. Не получены данные о возможной связи ожирения с риском ХП, наоборот, показано, что частота ожирения у больных ХП не различается от распространенности в целом по России. Выявленные ассоциации ожирения и РПЖ обуславливают необходимость (с целью канцерпревенции) дальнейшего изучения роли ожирения и связанных с ним метаболических расстройств в процессах онкогенеза.

\section{ДОПОЛНИТЕЛЬНАЯ ИНФОРМАЦИЯ.}

Источник финансирования. Работа выполнена по Государственному заданию в рамках бюджетной темы № АAАA-А17-117112850280-2.

Конфликт интересов. Авторы заявляют об отсутствии явных и потенциальных конфликтов интересов, связанных с публикацией настоящей статьи.

Участие авторов. Григорьева И.Н. - концепция и дизайн исследования, анализ данных, написание текста; Ефимова О.В. - сбор и обработка материала, анализ данных, написание текста; Суворова Т.С. - сбор материала. Все авторы внесли значимый вклад в проведение исследования и подготовку статьи, прочли и одобрили финальную версию статьи перед публикацией.

\section{СПИСОК ЛИТЕРАТУРЫ | REFERENCES}

1. Григорьева И.Н. Острый и хронический панкреатит. Новосибирск: Наука, 2011. [Grigor'eva IN. Ostryy i khronicheskiy pankreatit. Novosibirsk: Nauka; 2011. (In Russ.)]

2. Дедов И.И., Мельниченко Г.А., Шестакова М.В., и др Национальные клинические рекомендации по лечению морбидного ожирения у взрослых. 3-ий пересмотр (лечение морбидного ожирения у взрослых) // Ожирение и метаболизм. - 2018. - Т. 15. - № 1 - С. 53-70. [Dedov II, Melnichenko GA, Shestakova MV, et al. Russian national clinical recommendations for morbid obesity treatment in adults. 3rd revision (Morbid obesity treatment in adults). Obesity and metabolism. 2018;15(1):53-70. (In Russ.)] doi: https://doi.org/10.14341/OMET2018153-70

3. Григорьева И.Н., Логвиненко Е.В., Веревкин Е.Г., и др. Клинические характеристики, фактическое питание и профилактика желчнокаменной болезни в сочетании с метаболическим синдромом у женщин // Экспериментальная и клиническая гастроэнтерология. 2019. - № 5. - C. 91-96. [Grigorieva IN, Logvinenko EV, Verevkin EG, et al. Clinical features, nutrition and prevention of gallstone disease in combination with metabolic syndrome in women. Experimental and clinical gastroenterology. 2019;165(5):91-96. (In Russ.)] doi: https://doi.org/10.31146/1682-8658-ecg-165-5-91-96 
4. Григорьева И.Н. Гипертриглицеридемический острый панкреатит: прогресс и проблемы // Атеросклероз. - 2017. - Т. 13. - № 1. C. 41-48. [Grigor'eva IN. Hypertriglyceridemic acute pancreatitis: progress and problems. Ateroskleroz. 2017;13(1):41-48. (In Russ.)]

5. Navina S, Acharya C, DeLany JP, et al. Lipotoxicity causes multisystem organ failure and exacerbates acute pancreatitis in obesity. Sci Trans/Med. 2011;3(107):107ra1 10. doi: https://doi.org/10.1126/scitransImed.3002573

6. Deenadayalu VP, Blaut U, Watkins JL, et al. Does obesity confer an increased risk and/or more severe course of post-ERCP pancreatitis: a retrospective, multicenter study. J Clin Gastroenterol. 2008;42(10):1103-1109. doi: https://doi.org/10.1097/MCG.0b013e318159cbd1

7. Mendez-Bailon M, de Miguel Yanes JM, Jimenez-Garcia $R$, et al. National trends in incidence and outcomes of acute pancreatitis among type 2 diabetics and nondiabetics in Spain (2001-2011). Pancreatology. 2015;15(1):64-70. doi: https://doi.org/10.1016/j.pan.2014.11.004.

8. Acharya C, Cline RA, Jaligama D, et al. Fibrosis reduces severity of acute-on-chronic pancreatitis in humans. Gastroenterology. 2013;145(2):466-475. doi: https://doi.org/10.1053/j.gastro.2013.05.012

9. Дмитриев А.Н. Метаболический синдром: маркеры индивидуальной предрасположенности, диагностика доклинической стадии, обоснование тактики ведения пациентов // Международный эндокринологический журнал. - 2011. - Т. 2. № 34. - C. 93-102. [Dmitriev AN. Metabolicheskiy sindrom: markery individual'noy predraspolozhennosti, diagnostika doklinicheskoy stadii, obosnovanie taktiki vedeniya patsientov. International journal of endocrinology. 2011;2(34):93-102. (In Russ.)]

10. Larsson SC, Orsini N, Wolk A. Body mass index and pancreatic cancer risk: A meta-analysis of prospective studies. Int $\mathrm{J}$ Cancer 2007;120(9):1993-1998. doi: https://doi.org/10.1002/ijc.22535

11. Lin Y, Kikuchi S, Tamakoshi A, et al. JACC Study Group. Obesity, physical activity and the risk of pancreatic cancer in a large Japanese cohort. Int J Cancer. 2007;120(12):2665-2671. doi: https://doi.org/10.1002/ijc.22614

12. Tsang NM, Pai PC, Chuang CC, et al. Overweight and obesity predict better overall survival rates in cancer patients with distant metastases. Cancer Med. 2016;5(4):665-675. doi: https://doi.org/10.1002/cam4.634

13. Lennon H, Sperrin M, Badrick E, et al. The Obesity Paradox in Cancer: a Review. Curr Oncol Rep. 2016;18(9):56. doi: https://doi.org/10.1007/s11912-016-0539-4

14. World Cancer Research Fund International. Diet, nutrition, physical activity, and breast cancer survivors. 2014

15. Румянцев П.О., Саенко В.А., Румянцева У.В., Чекин С.Ю. Статистические методы анализа в клинической практике. Часть. 2. Анализ выживаемости и многомерная статистика // Проблемы эндокринологии. - 2009. - Т. 55. - № 6. - С 48-56. [Rumyantsev PO, Saenko VA, Rumyantseva UV, Chekin SY. Statistical methods of analysis in clinical practice. Part. 2. Survival analysis and multivariate statistics. Problems of endocrinology. 2009;55(6):48-56. (In Russ.)] doi: https://doi.org/10.14341/probl200955648-56

16. Муромцева Г.А., Концевая А.В., Константинов В.В., и др. Распространенность факторов риска неинфекционных заболеваний в российской популяции в 2012-2013 гг. Результаты исследования ЭССЕ-РФ // Кардиоваскулярная терапия и профилактика. - 2014. - Т.13. - №6. - С.4-11. [Muromtseva GA, Kontsevaya AV, Konstantinov W, et al. The prevalence of non-infectious diseases risk factors in Russian population in 2012-2013 years. The results of ECVD-RF Cardiovascular therapy and prevention. 2014;13(6):4-11. (In Russ.)] doi: https://doi.org/10.15829/1728-8800-2014-6-4-11

17. Chen SM, Xiong GS, Wu SM. Is obesity an indicator of complications and mortality in acute pancreatitis? An updated meta-analysis. J Dig Dis. 2012;13(5):244-251. doi: https://doi.org/10.1111/j.1751-2980.2012.00587.x

18. Martínez J, Sánchez-Payá J, Palazón JM, et al. Is obesity a risk factor in acute pancreatitis? A meta-analysis. Pancreatology. 2004;4(1):42-48. doi: https://doi.org/10.1159/000077025

19. Wang SQ, Li SJ, Feng QX. Overweight is an additional prognostic factor in acute pancreatitis: a meta-analysis. Pancreatology. 2011;11(2):92-98. doi: https://doi.org/10.1159/000327688
20. Al-Azzawi HH, Wade TE, Swartz-Basile DA, et al. Acute pancreatitis in obesity: adipokines and dietary fish oil. Dig Dis Sci. 2011;56(8):2318-2325. doi: https://doi.org/10.1007/s10620-011-1626-x

21. Hall TC, Stephenson JS, Jones MJ, et al. Is Abdominal Fat Distribution Measured by Axial CT Imaging an Indicator of Complications and Mortality in Acute Pancreatitis? J Gastrointest Surg. 2015;19(12):2126-2131. doi: https://doi.org/10.1007/s11605-015-2972-3

22. Taguchi M, Kubo T, Yamamoto M, et al. Body mass index influences the outcome of acute pancreatitis: an analysis based on the Japanese administrative database. Pancreas. 2014;43(6):863-866. doi: https://doi.org/10.1097/MPA.00000000000000137

23. Ammann RW, Raimondi S, Maisonneuve P, et al. Is obesity an additional risk factor for alcoholic chronic pancreatitis? Pancreatology. 2010;10(1):47-53. doi: https://doi.org/10.1159/000225921

24. Машарипова Ю.К., Шамсутдинова М.И. Хронический панкреатит: цитокины и клиническое течение // Вестник науки и образования. - 2019. - № 19-3. - С. 49-52. [Masharipova YK, Shamsutdinova MI. Chronic pancreatitis: cytokines and clinical course. Vestnik nauki l obrazovaniya. 2019;(19-3):49-52. (In Russ.)]

25. Olesen SS, Frandsen LK, Poulsen JL, et al. The prevalence of underweight is increased in chronic pancreatitis outpatients and associates with reduced life quality. Nutrition. 2017;43-44:1-7. doi: https://doi.org/10.1016/j.nut.2017.06.019

26. Roberts KM, Golian P, Nahikian-Nelms M, et al. Does the Healthy Eating Index and Mediterranean Diet Score Identify the Nutritional Adequacy of Dietary Patterns in Chronic Pancreatitis? Dig Dis Sci. 2019;64(8):2318-2326. doi: https://doi.org/10.1007/s10620-019-05536-3

27. Гаврилина Н.С., Ильченко Л.Ю., Седова Г.А., и др. Коррекция трофологической недостаточности у больных хроническим панкреатитом // Архивъ внутренней медицины. - 2019. T. 9. - № 1. - C. 70-84. [Gavrilina NS, Ilchenko LY, Sedova GA, et al. Correction of trophic insufficiency in patients with chronic pancreatitis. Russian archives of internal medicine. 2019:9(1):70-84. (In Russ.)] doi: https://doi.org/10.20514/2226-6704-2019-9-1-70-80

28. Губергриц Н.Б., Бондаренко О.А. Показатели ультразвуковой гистографии поджелудочной железы в динамике больных с хроническим панкреатитом на фоне ожирения // Гастроентерология. - 2013. - № 2. - С. 64-67. [Gubergrits NB, Bondarenko OA. Indicators of ultrasonic histography of the pancreas in the dynamics of treatment of patients with chronic pancreatitis on the background of obesity. Gastroenterology. 2013;(2):64-67. (In Russ.)]

29. Johansen $D$, Stocks $T$, Jonsson $H$, et al. Metabolic factors and the risk of pancreatic cancer: a prospective analysis of almost 580,000 men and women in the Metabolic Syndrome and Cancer Project. Cancer Epidemiol Biomarkers Prev. 2010;19(9):2307-2317. doi: https://doi.org/10.1158/1055-9965.EPI-10-0234

30. Luo J, Iwasaki M, Inoue M, et al. Body mass index, physical activity and the risk of pancreatic cancer in relation to smoking status and history of diabetes: a large-scale population-based cohort study in Japan--the JPHC study. Cancer Causes Control. 2007;18(6):603-612. doi: https://doi.org/10.1007/s10552-007-9002-z

31. Genkinger JM, Kitahara CM, Bernstein L, et al. Central adiposity, obesity during early adulthood, and pancreatic cancer mortality in a pooled analysis of cohort studies. Ann Oncol. 2015;26(11):2257-2266. doi: https://doi.org/10.1093/annonc/mdv355

32. Shi YQ, Yang J, Du P, et al. Effect of Body Mass Index on Overall Survival of Pancreatic Cancer A Meta-Analysis. Medicine (Baltimore). 2016;95(14):e3305. doi: https://doi.org/10.1097/MD.0000000000003305

33. Majumder K, Gupta A, Arora N, et al. Premorbid Obesity and Mortality in Patients with Pancreatic Cancer: A Systematic Review and Meta-analysis. Clin Gastroenterol Hepatol. 2016;14(3):355-e32. doi: https://doi.org/10.1016/j.cgh.2015.09.036.

34. World Cancer Research Fund A. Food, Nutrition, Physical Activity, and the Prevention of Cancer: a Global Perspective. Washington: American Institute for Cancer Research. 2007. 


\section{ИНФОРМАЦИЯ ОБ АВТОРАХ [AUTHORS INFO]:}

*Ефимова Ольга Васильевна, аспирант [Olga V. Efimova, postgraduate student]; адрес: Россия, 630089, Новосибирск, ул. Бориса Богаткова, д. 175/1 [address: 175/1, B. Bogatkova street, Novosibirsk, 630089, Russia]; ORCID: https://orcid.org/0000-0003-1874-8458; eLibrary SPIN: 7447-9338; e-mail: kukisyak@mail.ru.

Григорьева Ирина Николаевна, д.м.н., професcop [Irina N. Grigor'eva, MD, PhD, professor]; ORCID: https://orcid.org/0000-0003-0069-7744; eLibrary SPIN: 7198-3163; e-mail: igrigorieva@ngs.ru Суворова Татьяна Станиславовна, К.M.н. [Tatyana S. Suvorova, MD, PhD];

ORCID: https://orcid.org/0000-0001-5809-2241; SPIN: eLibrary 2450-1156; e-mail: tatyana.suvorova13@yandex.ru

*Автор, ответственный за переписку / Corresponding author.

\section{ЦИТИРОВАТЬ:}

Григорьева И.Н., Ефимова О.В., Суворова Т.С. Частота ожирения у больных острым панкреатитом, хроническим панкреатитом и раком поджелудочной железы // Ожирение и метаболизм. - 2020. - Т. 17. - №2. - С. 171-178. doi: https://doi.org/10.14341/omet12329

\section{TO CITE THIS ARTICLE:}

Grigor'eva IN, Efimova OV, Suvorova TS. The frequency of obesity in patients with acute pancreatitis, chronic pancreatitis and pancreatic cancer. Obesity and metabolism. 2020;17(2):171-178. doi: https://doi.org/10.14341/omet12329 